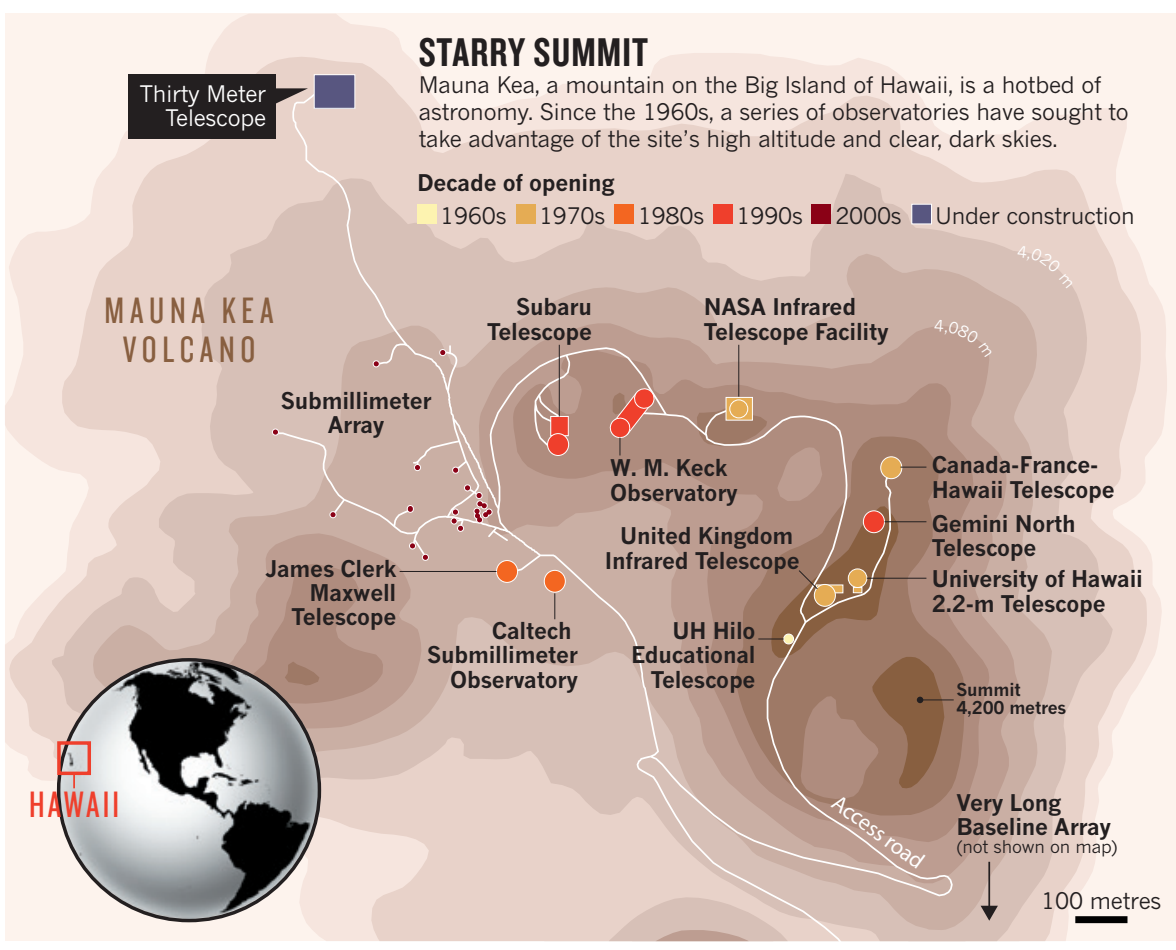

- university owns a 2.2-metre optical telescope that is the oldest on Mauna Kea, dating back to 1970; a 0.9-metre educational optical telescope; and the 3.8-metre United Kingdom Infrared Telescope (UKIRT). It also manages the 3-metre Infrared Telescope Facility for NASA, which studies planets, asteroids and stars.

"We have always made the point that space on the top of the mountain should only be populated by the absolutely best telescopes," says Günter Hasinger, director of the university's Institute for Astronomy.

\section{EYES SHUT}

The first to go will be the Caltech Submillimeter Observatory, the closure of which was announced in 2009. It will end operations in September, and then will be dismantled. Other telescopes, including Keck, Gemini and Subaru, involve complex international agreements that cannot be overwritten by the state of Hawaii alone. All have committed to operating on the mountain to the end of 2033.

"We intend to continue operating until we come to a point where the science return isn't worth it," says Raymond Blundell, an astronomer at the Harvard-Smithsonian Center for Astrophysics in Cambridge, Massachusetts, and director of the Submillimeter Array, an eight-dish radio telescope array on Mauna Kea.

Some of the telescopes on the mountain have just begun a new lease of life. Earlier this year, a consortium of east Asian observatories took over the submillimetre-wavelength
James Clerk Maxwell Telescope to study how galaxies and stars form, among other things. And UKIRT has just begun a long-term science programme that involves studying space debris and near-Earth asteroids, says director Richard Green, an astronomer at the University of Arizona in Tucson.

For now, Green continues to plan for nearly two decades ahead - although he acknowledges that the situation may change. "We realize there has to be more attention paid to the culture and how the mountain is taken care of," he says.

In addition to closing telescopes, Ige levied a list of other requirements. When the University of Hawaii's lease ends in 2033, it must return to state protection more than 40 square kilometres of the 45 it leases. Visitors to the summit must receive cultural training. And the TMT location, which is a few hundred metres beneath the actual summit, will be the last area on Mauna Kea on which any telescope will ever be built.

Nearly every telescope project on Mauna Kea in recent years has faced local protests, although not the sustained high emotion inspired by the TMT. John Johnson, an astronomer at Harvard University in Cambridge, says that astronomers should not be on the mountain top at all, given the history of the Hawaiian Islands. "This goes way beyond whether we construct this telescope or not," he says. "It has to do with the fact that the United States stole Hawaii from a sovereign people and proceeded to systematically erase that culture."

The university says it will have a plan for removing $25 \%$ of the observatories by the end of this year. The TMT has not announced whether and when it will resume construction, and legal challenges to the project are still wending their way through Hawaiian courts.

Two competing next-generation telescopes are being planned for Chile.

\section{BY ELIZABETH GIBNEY}

$\mathrm{H}$ appy birthday, caesium clock. Now move over. As the atomic clock used to define time itself turns 60 , tests are set to begin on a new generation of clocks that are designed to give the caesium version a run for its money.

Such timekeepers would enable a variety of experiments, including testing whether the fundamental constants of nature really are constant over time, and, eventually, a more precise official definition of the second.

Atomic clocks track the frequency of electromagnetic waves emitted by atoms as they change energy states. First demonstrated by British physicist Louis Essen in June 1955, the caesium clock became the world's official timekeeper in 1967 - defining the second as the time it takes for the microwaves that are absorbed or emitted when caesium atoms switch between states to cycle through $9,192,631,770$ oscillations.

Over the past decade, various laboratories have created prototype optical atomic clocks, which use different elements such as strontium and ytterbium that emit and absorb 
higher-frequency photons in the visible spectrum. This finer slicing of time should, in principle, make them more accurate: it is claimed that the best of these clocks gain or lose no more than one second every 15 billion years $\left(10^{18}\right.$ seconds $)$ - longer than the current age of the Universe - making them 100 times more precise than their caesium counterparts. Optical clocks are claimed to be the best timekeepers in existence, but the only way to verify this in practice is to compare different models against each other and see whether they agree.

Starting on 4 June, four European laboratories will kick off this testing process the National Physical Laboratory (NPL) in Teddington, UK; the department of TimeSpace Reference Systems at the Paris Observatory; the German National Metrology Institute (PTB) in Braunschweig, Germany; and Italy's National Institute of Metrology Research in Turin. Between them, the labs host a variety of optical clocks that harness different elements in different experimental set-ups.

For the first test, each institute will transmit a signal related to the optical frequency of its clocks to a satellite, which will beam the frequencies back down to the other labs. This will allow the labs to compare the frequencies of light emitted by their clocks and thus measure whether they all keep time to the same beat.

"It's really exciting," says Andrew Ludlow, a physicist at an optical-clock powerhouse run by the US National Institute of Standards and Technology (NIST) in Boulder, Colorado, who is not involved in the project. "A couple of comparisons of optical clocks have been made before, but on nothing like this scale." With more clocks, it should be easier to root out the source of any discrepancies, adds Helen Margolis, a physicist at the NPL.

She notes that a higher frequency does not necessarily mean a more accurate clock, factors can affect the ability of different clocks to keep time in practice. The hope is that all the clocks will agree, suggesting that they are as precise as claimed. If some clocks do not, it will indicate that improvements are needed.

The initial test is only a prelude to a more accurate test, however, because it has one big limitation: to beam light to a satellite, it must be converted to a microwave frequency which means that much of the potential extra accuracy gained by using visible light is lost. because varying sensitivities to environmental

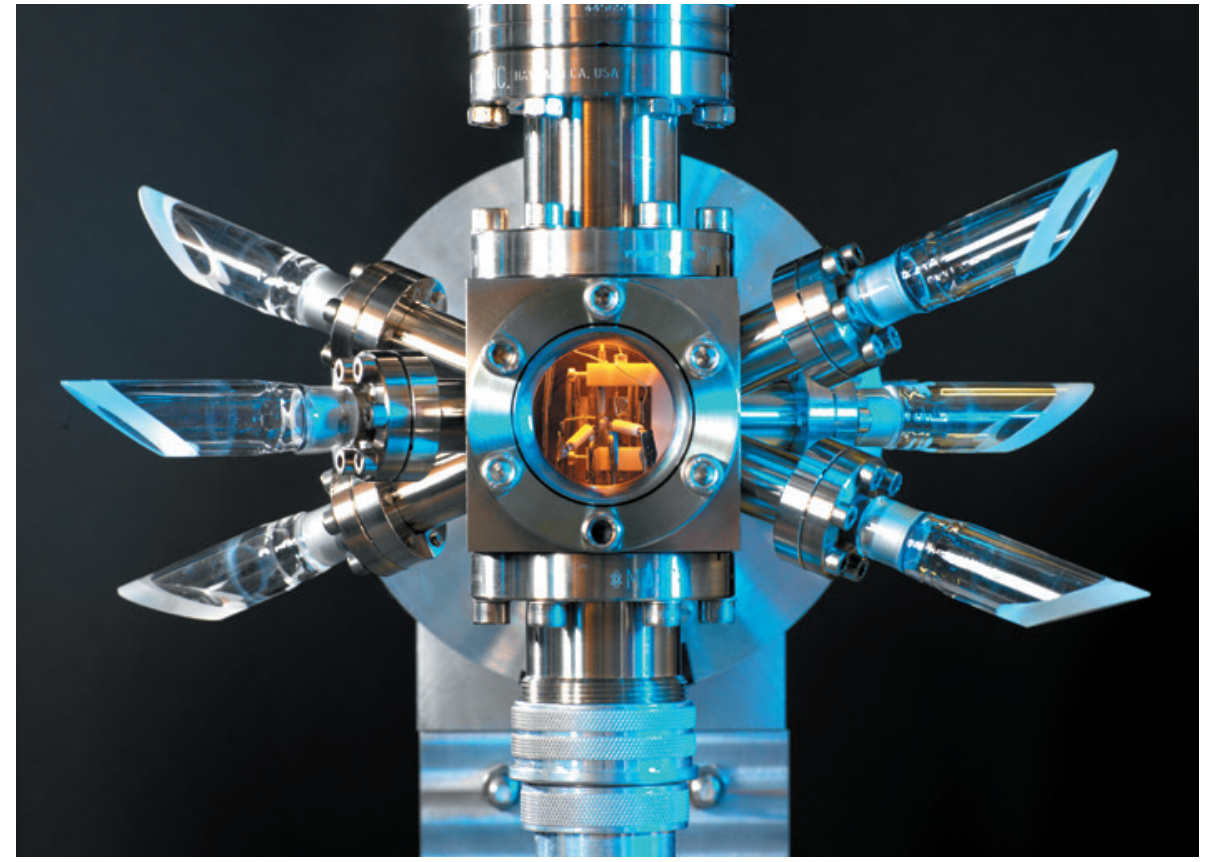

A strontium-ion optical clock housed at the National Physical Laboratory in Teddington, UK.
By increasing the rate of data transfer, the European labs hope to improve the accuracy of current state-of-the-art satellite comparisons by ten, but it will still be limited to one part in $10^{16}$. So the main function of the satellite test is to build confidence in optical clocks and show that they perform at least as well as existing caesium clocks, say researchers.

The more accurate test will transmit signals in

"We all think
our clocks
have a very
good potential
for achieving
the highest
accuracy."
the visible spectrum through fibre-optic cables to the labs. This will allow the clocks to be compared with an accuracy similar to the expected accuracies of the clocks themselves. Some of the labs have already established such links, and tests have begun on sections between Paris and Teddington, and Paris and Braunschweig. "Eventually, this would allow a four-way comparison. That's the vision," says Margolis.

"There is friendly competition," she adds. "We all think our clocks have a very good potential for achieving the highest accuracy or we wouldn't be working on them."

Fibre-optic links between optical atomic clocks already exist elsewhere, such as between the NIST lab and its partner lab JILA, also in Boulder. But these span shorter distances than the European network and are mostly between just two labs. "Europe is in a unique position as it has a high density of the best clocks in the world," says Fritz Riehle, a physicist at PTB.

Even if the clocks pass this later test, usurping the caesium clock to create a more precise definition of the second will not be easy. International atomic time - on which coordinated universal time, or UTC, is based - is currently calculated by averaging measurements from hundreds of atomic clocks. Doing the same with optical atomic clocks would require a way to aggregate time at this precise level; using the fibre-optic method across oceans is not currently feasible.

In the meantime, ever more precise time is important for improving global positioning systems, high-resolution radio astronomy and the time-stamping of financial transactions, as well as spotting tiny variations in fundamental constants. "Most attempts to unify gravity with other forces would lead to variations of fundamental constants in the expanding universe," says Marianna Safronova, a theorist at the University of Delaware in Newark.
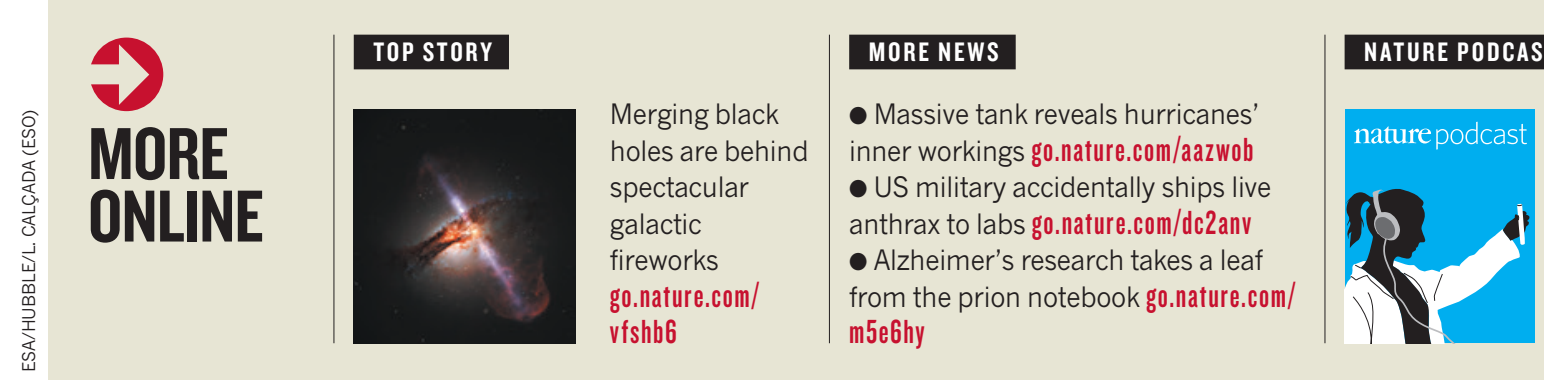

How immune cells escape the brain, what, and the case of the disappearing lake nature.com/ nature/podcast who's CRISPR-ing 